\title{
Phenolic compounds and anticancer activity of commercial sugarcane cultivated in Brazil
}

\author{
VANESSA G. ALVES ${ }^{1}$, ALAN G. SOUZA ${ }^{1}$, LUCAS U.R. CHIAVELLI ${ }^{1}$, ANA L.T.G. RUIZ ${ }^{2}$, \\ JOÃO E. CARVALHO ${ }^{2}$, ARMANDO M. POMINI ${ }^{1}$ and CLEUZA C. SILVA ${ }^{1}$
}

\author{
${ }^{1}$ Universidade Estadual de Maringá/UEM, Departamento de Química, Avenida Colombo, 5790, 87020-900 Maringá, PR, Brasil \\ ${ }^{2}$ Universidade Estadual de Campinas, CPQBA, Instituto de Química, Rua \\ Alexandre Cazellato, 999, 13081-970 Paulínia, SP, Brasil
}

Manuscript received on May 19, 2015; accepted for publication on December 8, 2015

\begin{abstract}
The cultivation of sugarcane hybrids (X Saccharum officinarum L.) is an important revenue source for the Brazilian economy. Herein it is reported the evaluation of the cytotoxic activity of mid-polarity sugarcane extracts against human cancer cell lines, as well as the isolation of steroids sitosterol, stigmasterol and campesterol, phenolic acids $p$-hydroxybenzoic, $p$-hydroxycinnamic, vanillic and ferulic acid, terpenoids $\alpha$-tocopherol and $\beta$-carotene and a novel substance in sugarcane, the flavonoid aglycone tricin (5,7,4-trihydroxy-3,5-dimethoxyflavone). The presence of large amounts of phenolic acids and the flavonoid tricin may explain the cytostatic activity observed for the mid-polarity crude extract and filtrates.
\end{abstract}

Key words: anticancer activity, phenolic compounds, Saccharum officinarum L., sugarcane.

\section{INTRODUCTION}

Sugarcane (Saccharum officinarum L., Poaceae), a grass native to Asia, is widely found in tropical regions and it is one of the most important cultures in Brazil, with an annual production of over 375 million tons (Conab 2013). It is widely used in sugar and alcohol production, yielding high quality at a low price for both products. Sugarcane is used to produce brown sugar bars (rapadura), molasses, rum (cachaça), in addition to its juice (garapa), which is largely consumed in Brazil.

Besides its great economic importance for food and fuel production, the literature reports medicinal properties for $S$. officinarum preparations, such

Correspondence to: Cleuza C. Silva

E-mail: ccsilva@uem.br as antidiabetic (Takahashi et al. 1985) and antimutagenicity (Yamashita et al. 1993) activities, as well as its use in osteoporosis treatment (Barros et al. 2007), usually associated with the juice prepared from the culms. The Ayurveda cites the use of this species to treat anemia, inflammations and ulcers (Chatterjee and Pakrashi 1995).

The most common phenolic compounds found in S. officinarum belong to the flavone class, such as naringenin, apigenin, tricin and luteolin derivatives (Williams et al. 1974, López-Lázaro 2009). These compounds are partly responsible for the sugarcane juice color and are acknowledged to have beneficial health effects associated with their high antioxidant potential and capacity to protect cells from degenerative processes and reduce the development of cancer and cardiovascular diseases. 
Phytochemical studies reported the presence and biological potential of these sugarcane compounds. A bioguided study of the juice from culms of $S$. officinarum led to isolation of the flavone tricin-7-O- $\beta$-(6'"-methoxycinnamic)glycoside, which has shown considerable antiproliferative and antioxidant potential (Almeida et al. 2007). Other studies have shown that extracts of sugarcane leaves could improve health quality, decreasing the incidence of diseases due to their antioxidant properties (Abbas et al. 2013). Other phytochemical studies reported the presence of flavonoid glycosides (Colombo et al. 2005, 2006a, b, Walford et al. 2009), wherever this class of natural products has been considered fundamental to human health.

In addition to the isolated flavonoids, other important bioactive compounds have been found in this species, such as hydroxamic acids, hydroxycinnamic acids, alpha hydroxy acids, aliphatic acids and polyamides (Loyd and Naidoo 1983). Alpha hydroxy acids are used in cosmetics for peeling (Ruciani 1997), in dermatology as a photoprotector and an anti-inflammatory (Perricone and Dinardo 1996). In the last years, the phenolic compounds of $S$. officinarum gained increasing attention regarding their antioxidant potential. The literature suggests the use of sugarcane as a dietary source of antioxidants, flavonoids like luteolin8-C-(rhamnosyl glucoside), diosmentin-8-Cglucoside, vitexin, schaftoside, isoschaftoside and 4',5'-dimethyl-luteolin-8-C-glucoside, the major compounds responsible for this activity (Vila et al. 2008). Recent studies have reported this property (Ahmad et al. 2011, Duarte-Almeida et al. 2011).

The presence of anthocyanins in the methanolic crude extract from $S$. officinarum was recently reported (Pallavi et al. 2012). The same study demonstrated that the highly polar crude extract has cytotoxic potential against HT29 human colon cancer cells.
Although the presence of bioactive substances in highly polar sugarcane extracts is well known, little is known about mid-polarity extract compounds and their anticancer potential. Therefore, the antiproliferative activity of the ethyl acetate extract and fractions and the isolation of chemical constituents from the leaves of $S$. officinarum commercially cultivated in Brazil are reported herein.

\section{MATERIALS AND METHODS}

\section{GENERAL EXPERIMENTAL PROCEDURES}

NMR spectra were obtained with a Varian spectrometer, model Mercury plus BB, operating at 300.06 MHz for ${ }^{1} \mathrm{H}$ and $75.5 \mathrm{MHz}$ for ${ }^{13} \mathrm{C}$. Chemical shifts were recorded in $\mathrm{ppm}$ with reference to internal tetramethylsilane (TMS $\delta=0.0 \mathrm{ppm}$ ). The solvents used were $\mathrm{CDCl}_{3}$ and $\mathrm{CD}_{3} \mathrm{OD}$ (Aldrich). Column chromatography (CC) was performed using silica gel 60 (Merck) or Sephadex LH-20 (Sigma). For thin layer chromatography (TLC), either silica gel $60 \mathrm{G}$ or 60 GF254 (Merck) were employed. TLC spots were visualized by spraying an acetic acid $/ \mathrm{H}_{2} \mathrm{SO}_{4}$ /anysaldehyde solution (1:0.5:48.5 mL) followed by plate heating.

Gas chromatography coupled to mass spectrometry analyses were performed in a Focus GC (Thermo Finnigan) gas chromatograph coupled to a DSQ II (Thermo Finnigan) mass selective detector working with $70 \mathrm{eV}$ electron impact, fitted with a quadrupole type analyzer and an electron impact detector (FID). High-purity helium was used as mobile phase with a column flow of $1 \mathrm{~mL} \mathrm{~min}^{-1}$. The analyses were performed with the injector working at $250{ }^{\circ} \mathrm{C}$ and splitless injection. The capillary column used was DB-5 (30m x $0.25 \mathrm{~mm} \times 0.25 \mu \mathrm{m}$ ) and the oven temperature program was from $100{ }^{\circ} \mathrm{C}$ to $290^{\circ} \mathrm{C}$ at $10{ }^{\circ} \mathrm{C} \mathrm{min}$. Sample aliquots were prepared from $1 \mathrm{mg}$ of each compound dissolved in $1 \mathrm{~mL}$ of ethyl acetate (HPLC grade). 
SUGARCANE SAMPLES, PREPARATION AND EXTRACT PURIFICATION

Saccharum officinarum samples were harvested from a commercial culture in the city of Munhoz de Mello, Paraná, Brazil. The material (4.36 kg) was crushed and exhaustively extracted with ethyl acetate at room temperature, yielding $72.00 \mathrm{~g}$ of crude extract. The extract was submitted to partition on a silica gel column eluted with hexane $(7.64 \mathrm{~g})$, ethyl acetate (33.75 g) and methanol (7.72 g).

ISOLATION OF CHEMICAL CONSTITUENTS

Part of the hexane partition (1.0 g) was submitted to silica gel column chromatography with hexane, chloroform and methanol in increasing polarity gradient. This procedure yielded $\beta$-carotene (1, $3.9 \mathrm{mg})$.

Silica gel column chromatography of the ethyl acetate partition $(32.00 \mathrm{~g})$ with hexane, hexane:dichloromethane, dichloromethane, dichloromethane:ethyl acetate, ethyl acetate, ethyl acetate:methanol and methanol in increasing polarity gradient yielded 548 fractions. The fractions eluted with $\mathrm{CH}_{2} \mathrm{Cl}_{2}$ :AcOEt (85:15) were combined and washed with ethyl acetate, leading to the isolation of flavonoid tricin $(8,55.0 \mathrm{mg})$. The fractions eluted with $\mathrm{CH}_{2} \mathrm{Cl}_{2}$ :AcOEt $(3: 2)$ were combined, washed with cold propanone and yielded compound sitosterol glucoside (9, 104.2 $\mathrm{mg}$ ).

Similarly, part of the fractions eluted with $\mathrm{CH}_{2} \mathrm{Cl}_{2}$ :AcOEt (9:1) were combined and purified in Sephadex LH 20 with methanol as eluent. This procedure led to the isolation of a mixture of $p$-hydroxycinnamic and $p$-hydroxybenzoic acids (10 and 11, $33.5 \mathrm{mg}$ ). The fractions eluted with $100 \%$ dichloromethane were combined and washed with ethyl acetate and methanol and yielded a mixture of steroids sitosterol, stigmasterol and campesterol (2-4, $30.0 \mathrm{mg})$. After, the remaining dough of fractions combined $(2.08 \mathrm{~g})$ was submitted to silica gel column chromatography with hexane, chloroform and methanol in increasing polarity gradient yielding the compound $\alpha$-tocopherol (5, $3.6 \mathrm{mg})$. The fractions eluted with $\mathrm{HCCl}_{3}: \mathrm{MeOH}$ $20 \%$ were combined and purified in silica gel column chromatography with hexane, ethyl acetate and methanol in increasing polarity gradient. This procedure yielded compounds vanillic acid $(\mathbf{6}, 6.0$ $\mathrm{mg})$ and ferulic acid (7, $5.0 \mathrm{mg})$.

SPECTRAL DATA OF THE COMPOUNDS 1-11 OBTAINED IN THIS WORK

Sitosterol (2). GC-MS (EI, $70 \mathrm{eV}) \mathrm{m} / \mathrm{z}: 414.31\left(\mathrm{M}^{+}\right.$ 1), 55.00 (100), 69.01 (93.3), 83.05 (79.8), 80.99 (76.0 ). ${ }^{1} \mathrm{H}$ NMR $\left(300.06 \mathrm{MHz}, \mathrm{CDCl}_{3}\right) . \delta 3.47(1 \mathrm{H}$, m, H-3), 5.36 (1H, d; 5.1, H-6), 0.68 (3H, s, H-18), 1.01 (3H, s, H-19), 0.93 (3H, s, H-21), 0.84 (3H, s, $\mathrm{H}-26), 0.81$ (3H, s, H-27), 0.87 (3H, s, H-29). ${ }^{13} \mathrm{C}$ NMR (75.5 MHz, $\mathrm{CDCl}_{3}$ ): $\delta 37.4(\mathrm{C}-1), 31.8(\mathrm{C}-$ 2), 72.0 (C-3), 42.5 (C-4), 140.9 (C-5), 121.9 (C6), 31.8 (C-7), 32.1 (C-8), 50.3 (C-9), 36.3 (C-10), 21.2 (C-11), 39.8 (C-12), 42.5 (C-13), 56.9 (C-14), 24.5 (C-15), 28.4 (C-16), 56.2 (C-17), 12.0 (C-18), 19.6 (C-19), 36.1 (C-20), 19.6 (C-21), 33.9 (C-22), 39.2 (C-23), 46.0 (C-24), 29.1 (C-25), 18.9 (C-26), 19.6 (C-27), 23.2 (C-28), 12.2 ppm (C-29).

Stigmasterol (3). GC-MS (EI, $70 \mathrm{eV}) \mathrm{m} / \mathrm{z}$ : $412.26\left(\mathrm{M}^{+}\right.$2), 43.02 (100), 105.01 (84.9), 95.05 (84.6), 107.03 (93.59). ${ }^{1} \mathrm{H}$ NMR (300.06 MHz, $\left.\mathrm{CDCl}_{3}\right) . \delta 3.47(1 \mathrm{H}, \mathrm{m}, \mathrm{H}-3), 5.36(1 \mathrm{H}, \mathrm{d} ; 5.1$, H-6), 4.97 (1H, dd; 8.4, H-22), 5.19 (1H, dd; 8.4, H-23), 0.68 (3H, s, H-18), 1.01 (3H, s, H-19), 0.93 (3H, s, H-21), 0.84 (3H, s, H-26), 0.81 (3H, s, $\mathrm{H}-27), 0.87$ (3H, s, H-29). ${ }^{13} \mathrm{C}$ NMR (75.5 MHz, $\left.\mathrm{CDCl}_{3}\right): \delta 37.4(\mathrm{C}-1), 31.8(\mathrm{C}-2), 72.0$ (C-3), 42.5 (C-4), 140.9 (C-5), 121.9 (C-6), 31.8 (C-7), 32.1 (C-8), 50.3 (C-9), 36.3 (C-10), 21.2 (C-11), 39.8 (C-12), 42.5 (C-13), 56.9 (C-14), 24.5 (C-15), 28.4 (C-16), 56.1 (C-17), 12.0 (C-18), 19.6 (C-19), 40.7 (C-20), 21.4 (C-21), 138.5 (C-22), 129.4 (C-23), 51.4 (C-24), 31.8 (C-25), 19.2 (C-26), 19.2 (C-27), 25.6 (C-28), 12.4 ppm (C-29). 
Campesterol (4). GC-MS (EI, $70 \mathrm{eV}) \mathrm{m} / \mathrm{z}$ : $400.28\left(\mathrm{M}^{+} 71.7\right), 43.97$ (100), 43.03 (60.9), 145.07 (45.0), 95.06 (39.9). ${ }^{1} \mathrm{H}$ NMR (300.06 $\left.\mathrm{MHz}, \mathrm{CDCl}_{3}\right) . \quad \delta 3.47(1 \mathrm{H}, \mathrm{m}, \mathrm{H}-3), 5.36(1 \mathrm{H}, \mathrm{d}$; 5.1, H-6), 0.68 (3H, s, H-18), 1.01 (3H, s, H-19), $0.93(3 \mathrm{H}, \mathrm{s}, \mathrm{H}-21), 0.84$ (3H, s, H-26), $0.81(3 \mathrm{H}$, s, H-27), 0.78 (3H, s, H-28). ${ }^{13} \mathrm{C}$ NMR (75.5 MHz, $\mathrm{CDCl}_{3}$ ). $\delta 37.4$ (C-1), 31.8 (C-2), 72.0 (C-3), 42.5 (C-4), 140.9 (C-5), 121.9 (C-6), 31.8 (C-7), 32.1 (C-8), 50.3 (C-9), 36.3 (C-10), 21.2 (C-11), 39.9 (C-12), 42.5 (C-13), 56.9 (C-14), 24.5 (C-15), 28.4 (C-16), 56.2 (C-17), 12.0 (C-18), 19.6 (C-19), 36.1 (C-20), 18.9 (C-21), 33.9 (C-22), 29.1 (C-23), 56.1 (C-24), 29.6 (C-25), 18.9 (C-26), 19.6 (C-27), 18.9 ppm (C-28).

Tricin (8). GC-MS (EI, $70 \mathrm{eV}$ ) m/z: 329.91 ( $\left.\mathrm{M}^{+} 100\right), 152.90$ (11.4), 43.98 (9.8), 151.04 (8.45), 206.92 (7.44). ${ }^{1} \mathrm{H} \mathrm{NMR}\left(300.06 \mathrm{MHz}, \mathrm{CD}_{3} \mathrm{OD} /\right.$ $\mathrm{CDCl}_{3}$ ). $\delta 7.19$ (s, H-2' and 6'), 6.60 (s, H-3), 6.23 (d; 2.1, H-6), 6.46 (d; 2.1, H-8), 3.98 ppm (s, $3^{\prime}-\mathrm{OCH}_{3}$ and 5 ' $\left.-\mathrm{OCH}_{3}\right) \cdot{ }^{13} \mathrm{C} \mathrm{NMR}(75.45 \mathrm{MHz}$, $\mathrm{CD}_{3} \mathrm{OD} / \mathrm{CDCl}_{3}$ ). $\delta 165.3$ (C2), 104.9 (C-3), 183.1 (C-4), 162.4 (C-5), 99.0 (C-6), 164.9 (C-7), 94.8 (C-8), 158.2 (C-9), 104.8 (C-10), 104.4 (C-2'), 148.7 (C-3'), 140.2 (C-4'), 148.7 (C-5'), 104.4 (C6'), 56.0 ppm (3'- $\mathrm{OCH}_{3}$ and 5' $\left.-\mathrm{OCH}_{3}\right)$.

p-hydroxycinnamic acid (10). GC-MS (EI, 70 eV) $m / z$ : $163.98\left(\mathrm{M}^{+} 100\right), 163.99$ (41.5), 162.97 (39.8), 118.99 (25.5), 117.97 (23.3). ${ }^{1} \mathrm{H}$ NMR $\left(300.06 \mathrm{MHz}, \mathrm{CD}_{3} \mathrm{OD} / \mathrm{CDCl}_{3}\right) . \delta 7.45(2 \mathrm{H}, \mathrm{dt} ; J=$ 8.4 e $4.8 \mathrm{~Hz}, \mathrm{H}-2$ e H-6) 6.81 (2H, dt; $J=8.4$ e 4.8 Hz, H-3 e H-5), 6.30 (1H, d; $J=15$ Hz; H-8), 7.62 ppm $(1 \mathrm{H}, \mathrm{d} ; J=15 \mathrm{~Hz} ; \mathrm{H}-7) .{ }^{13} \mathrm{C}$ NMR $(75.5 \mathrm{MHz}$, $\left.\mathrm{CD}_{3} \mathrm{OD} / \mathrm{CDCl}_{3}\right) . \delta 127.2(\mathrm{C}-1), 131.0(\mathrm{C}-2, \mathrm{C}-6)$, 116.0 (C-3, C-5), 161.1 (C-4), 146.6 (C-7), 115.6 $(\mathrm{C}-8), 170.0 \mathrm{ppm}(\mathrm{C}=\mathrm{O})$.

p-hydroxybenzoic acid (11). GC-MS (EI, 70 eV) $m / z: 137.94\left(\mathrm{M}^{+}\right.$5.1), 120.94 (100), 137.94 (70.3), 64.98 (16.4), 121.97 (7.9). NMR ${ }^{1} \mathrm{H}$ (300.06 $\left.\mathrm{MHz}, \mathrm{CD}_{3} \mathrm{OD} / \mathrm{CDCl}_{3}\right) .7 .90(2 \mathrm{H}, \mathrm{dt} ; J=8.4$ e 4.8 Hz, H-2 e H-6), 6.81 (2H, dt; $J=8.4$ e 4.8 Hz, H-3 e H-5). ${ }^{13} \mathrm{C}$ NMR (75.45 MHz, $\left.\mathrm{CD}_{3} \mathrm{OD} / \mathrm{CDCl}_{3}\right) . \delta$
131.0 (C-1), 132.9 (C-2, C-6), 116.7 (C-3, C-5), 163.3 (C-4), 171.0 ppm $(\mathrm{C}=\mathrm{O})$.

Ferulic acid (7). GC-MS (EI, $70 \mathrm{eV}) \mathrm{m} / \mathrm{z}$ : $193.97\left(\mathrm{M}^{+} 100\right), 43.97$ (20.6), 178.94 (19.4), 132.94 (17.5), 192.98 (11.3). ${ }^{1} \mathrm{H}$ NMR (300.06 $\left.\mathrm{MHz}, \mathrm{CD}_{3} \mathrm{OD}\right) \delta 7.17(1 \mathrm{H}, \mathrm{d}, 2.1 \mathrm{~Hz}, \mathrm{H}-2), 6.80$ $(1 \mathrm{H}, \mathrm{d} ; 8.4 \mathrm{~Hz}, \mathrm{H}-5) 7.06(1 \mathrm{H}, \mathrm{dd} ; 8.4$ e $2.1 \mathrm{~Hz}$, H-6), 7.58 (1H, d, 15.9 Hz, H-7), 6.33 (1H, d, 15.9 $\mathrm{Hz}), 3.87\left(\mathrm{OCH}_{3}\right) .{ }^{13} \mathrm{C} \mathrm{NMR}\left(75.45 \mathrm{~Hz}, \mathrm{CD}_{3} \mathrm{OD}\right), \delta$ 128.6 (C-1), 111.5 (C-2), 151.9 (C-3), 149.3 (C-4), 116.4 (C-5), 123.0 (C-6), 146.2 (C-7), 116.9 ( C-8), $56.4\left(\mathrm{OCH}_{3}\right), 171.0 \mathrm{ppm}$ ( C-9).

Vanillic acid (6). GC-MS (EI $70 \mathrm{eV}) \mathrm{m} / \mathrm{z}$ $167.95\left(\mathrm{M}^{+} 100\right), 152.98$ (80.1), 96.92 (25.8), 124.94 (21.6), 150.98 (18.3). ${ }^{1} \mathrm{H}$ NMR (300.06 $\left.\mathrm{MHz}, \mathrm{CD}_{3} \mathrm{OD}\right) \delta 7.54$ (2H, m, H-2 e H-6), 6.84 $(1 \mathrm{H}, \mathrm{d} ; 8.7 \mathrm{~Hz}, \mathrm{H}-5), 3.88 \mathrm{ppm}\left(3 \mathrm{H}, \mathrm{s}, \mathrm{OCH}_{3}\right) .{ }^{13} \mathrm{C}$ NMR (75.45 Hz, CD 3 OD) $\delta 128.4$ (C-1), 113.7 (C2), 151.9 (C-3), 152.7 (C-4), 115.8 (C-5), 125.5 (C-6), 170.0 (C-7), 56.4 ppm $\left(\mathrm{OCH}_{3}\right)$.

$\alpha$-tocopherol (5). GC-MS (EI, $70 \mathrm{eV}$ ) $\mathrm{m} / \mathrm{z}$ : $430.28\left(\mathrm{M}^{+} 70.8\right), 165.03$ (100), 164.03 (34.4), 166.04 (12.2), 205.04 (11.4). ${ }^{1} \mathrm{H}$ NMR (300.06 $\left.\mathrm{MHz}, \mathrm{CDCl}_{3}\right) . \delta 2.61(2 \mathrm{H}, \mathrm{t} ; 6.9, \mathrm{H}-4), 1.78(2 \mathrm{H}$, m, H-3), 2.17 (3H, s, H-8b), 2.12 (3H, s, H-7a), 1.59 (3H, s, H-5a), 1.26 (3H, s, H-2a), $0.88(3 \mathrm{H}$, s, H-4'a), 0.87 (3H, s, H-8'a), 0.85 (6H, s, H-12'a and $\mathrm{H}-13$ 'a) ${ }^{13} \mathrm{C}$ NMR (75.45 $\left.\mathrm{MHz}, \mathrm{CDCl}_{3}\right) \delta$ 145.7 (C-8a), 122.8 (C-8), 118.7 (C-7), 11.5 (C7a), 144.7 (C-6), 117.6 (C-5), 12.0 (C-5a), 121.9 (C-4a), 24.7 (C-4), 31.7 (C-3), 74.7 (C-2), 39.6 (C1'), 24.7 (C-2'), 37.3 (C-3', 4', 5', 7', 8', 9'), 25.0 (C-6'), 24.6 (C-10'), 39.9 (C-11'), 28.2 (C-12'), 22.9 (C-12'a, 13'), 19.9 (C-8'a, 4'a), 24.0 (C-2a), 12.4 ppm (C-8b).

Sitosterol glucoside (9). GC-MS (EI, $70 \mathrm{eV}$ ) m/z: $414.31\left(\mathrm{M}^{+} 0\right), 43.95$ (100), 206.93 (17.5), 43.01 (15.1), 164.96 (6.4). ${ }^{1} \mathrm{H}$ NMR (300.06 MHz, $\left.\mathrm{CD}_{3} \mathrm{OD} / \mathrm{CDCl}_{3}\right) . \delta 3.37(1 \mathrm{H}, \mathrm{m}, \mathrm{H}-3), 5.32(1 \mathrm{H}, \mathrm{d}$; 5.1, H-6), 0.67 (3H, s, H-18), 1.03 (3H, s, H-19), 0.92 (3H, s, H-21), 0.84 (3H, s, H-26), 0.83 (3H, s, H-27), 0.89 (3H, s, H-29), 4.40 (1H, d; 8.1, H-1'), 
3.53 (1H, m, H-2'), 3.46 (1H, m, H-3'), 3.35 (1H, m, H-4'), 3.70 (1H, m, H-5'), 3.54 (1H, m, H-6'a), 4.06 (1H, m, H-6'b). ${ }^{13} \mathrm{C}$ NMR (75.5 MHz, CD $\left.\mathrm{OD}\right)$

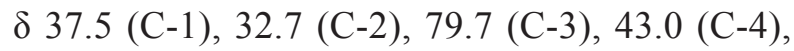
141.3 (C-5), 122.7 (C-6), 32.7 (C-7), 32.8 (C-8), 51.1 (C-9), 36.9 (C-10), 21.8 (C-11), 39.4 (C-12), 43.0 (C-13), 56.8 (C-14), 25.0 (C-15), 29.0 (C-16), 57.6 (C-17), 12.3 (C-18), 19.8 (C-19), 36.9 (C-20), 20.5 (C-21), 34.7 (C-22), 40.6 (C-23), 46.7 (C-24), 29.0 (C-25), 19.8 (C-26), 21.8 (C-27), 23.8 (C-28), 12.7 (C-29), 102.0 (C-1'), 74.5 (C-2'), 78.7 (C-3'), 71.1 (C-4'), 77.1 (C-5'), 62.5 ppm (C-6').

B-carotene (1). GC-MS (EI, $70 \mathrm{eV}) \mathrm{m} / \mathrm{z}: 536.97$ $\left(\mathrm{M}^{+}\right.$0), 43.99 (100), 43.04 (18.1), 55.02 (13.9), 57.04 (11.9). ${ }^{1} \mathrm{H}$ NMR (300.06 MHz, $\left.\mathrm{CDCl}_{3}\right)$. $\delta$ 2.02 (4H, m, H-4 and H-4'), 1.64 (4H, m, H-3 and H-3'), 1.46 (4H, m, H-2 and H-2'), 1.03 (6H, s, H-16 and H-16'), 1.04 (6H, s, H-17 and H-17'), 1.72 (6H, s, H-18 and H-18'), 1.97 (6H, s, H-19 and H-19'), 2.05 (6H, s, H-20 and H-20'). ${ }^{13} \mathrm{C}$ NMR (75.45 MHz, $\mathrm{CDCl}_{3}$ ) $\delta 34.5$ (C-1/1'), 39.9 (C-2/2'), 19.5 (C-3/3'), 33.3 (C-4/4'), 129.6 (C-5/5'), 137.9 (C-6/6'), 126.6 (C-7/7'), 137.5 (C-8/8'), 136.7 (C9/9'), 131.1 (C-10/10'), 125.2 (C-11/11'), 137.2 (C-12/12'), 136.7 (C-13/13'), 132.6 (C-14/14'), 129.6 (C-15/15'), 29.2 (C-16/16', 17/17'), 22.7 (C18/18'), 12.9 (C-19/19'), 12.9 ppm (C-20/20').

\section{In vitro ANTICANCER ASSAY}

The in vitro anticancer assays of the ethyl acetate extract and its hexane, ethyl acetate and methanol partitions and pure flavonoid tricin were performed as described in the literature (Monks et al. 1991). Eight human tumor cell lines [U251 (glioma), MCF7 (breast), NCI-ADR/RES (multiple drug resistant ovary cells), 786-0 (kidney), NCI-H460 (lung, non-small cells), PC-3 (prostate), OVCAR-03 (ovary) and colon (HT29)] were kindly provided by Frederick MA, National Cancer Institute/ USA. HaCat (human keratinocytes) cell line was kindly donated by Dr. Ricardo Della Coletta (FOP,
UNICAMP). Stock and experimental cultures were grown in media containing $5 \mathrm{~mL}$ RPMI 1640 (GIBCO BRL) supplemented with $5 \%$ fetal bovine serum (GIBCO BRL). A penicillin:streptomycin mixture $\left(1000 \mu \mathrm{g} \mathrm{mL}^{-1}: 1000 \mu \mathrm{g} \mathrm{mL}^{-1}, 1 \mathrm{~mL} \mathrm{~L}^{-1}\right.$ RPMI) was added to the experimental cultures. Cells plated to 96 -well plates $\left(100 \mu \mathrm{L}\right.$ cells well $\left.{ }^{-1}\right)$ were exposed to different sample concentrations in DMSO/RPMI $\left(0.25,2.5,25\right.$ and $\left.250 \mu \mathrm{g} \mathrm{mL}^{-1}\right)$ at $37{ }^{\circ} \mathrm{C}$ under $5 \% \mathrm{CO}_{2}$ in air for $48 \mathrm{~h}$. The final DMSO concentration did not affect cell viability. The cells were fixed with $50 \%$ trichloroacetic acid before $\left(\mathrm{T}_{0}\right.$ plate) and after ( $\mathrm{T}_{1}$ plates) sample addition. Cell proliferation was determined by spectrophotometric quantification $(540 \mathrm{~nm})$ of cell protein content using the sulforhodamine B assay. Using the concentration-response curve for each cell line, the $\mathrm{GI}_{50}$ (concentration resulting in growth inhibition of $50 \%$ ) was determined through nonlinear regression analysis in software ORIGIN 8.0 (OriginLab Corporation) (Shoemaker 2006).

\section{RESULTS AND DISCUSSION}

$\beta$-carotene (1), steroids sitosterol (2), stigmasterol (3) and campesterol (4), $\alpha$-tocopherol (5), vanillic (6) and ferulic acids (7), tricin (8), sitosterol glucoside (9) and $p$-hydroxycinnamic (10) and $p$-hydroxybenzoic acids (11) (Figure 1) were isolated from the ethyl acetate extract and identified by interpretation of ${ }^{1} \mathrm{H},{ }^{13} \mathrm{C}$, DEPT and COSY NMR spectral data and comparison with literature data (Miranda et al. 2012, Goulart et al. 1993, Yu et al. 2006, Sajjadi et al. 2012, Chung et al. 2005, Lee et al. 2007, Silva et al. 2001, Scott 1972).

The molecular formula of compound $\mathbf{8}$, $\mathrm{C}_{17} \mathrm{H}_{14} \mathrm{O}_{7}$, was established by GC-MS analysis $[\mathrm{m} / \mathrm{z}$ 329.91]. Its ${ }^{1} \mathrm{H}$ NMR spectrum presented signals characteristic of a substance belonging to the flavonoid class, of which the following stand out the singlet at $\delta_{\mathrm{H}} 7.19$ relative to hydrogens $\mathrm{H}-2$ ' and H-6' of ring B and doublets at $\delta_{\mathrm{H}} 6.46(J=2.1 \mathrm{~Hz})$ 
<smiles>[R]c1ccc(O)c(OC)c1</smiles><smiles>[R]C=CC(=O)O</smiles><smiles>COc1cc(-c2cc(=O)c3c(O)cc(O)cc3o2)cc(OC)c1O</smiles><smiles>[R]c1ccc(O)cc1</smiles>

$10 \mathrm{R}=$<smiles>[R]C=CC(=O)O</smiles>

$11 \mathrm{R}=$<smiles>O=C(O)F</smiles>

Figure 1 - Phenolic compounds isolated from sugarcane.

and $6.23(J=2.1 \mathrm{~Hz})$, corresponding to hydrogens $\mathrm{H}-8$ and $\mathrm{H}-6$ of ring $\mathrm{A}$, and a singlet at $\delta_{\mathrm{H}} 6.60$ attributed to hydrogen $\mathrm{H}-3$. The ${ }^{13} \mathrm{C}$ NMR spectrum showed resonances at $\delta_{\mathrm{C}} 158.6,94.8,164.9,99.6$, 162.2 and 104.9, attributed to carbons C-9, C-8, C-7, C-6, C-5 and C-10 of ring A, respectively. Furthermore, a signal at $\delta_{C} 183.1$, characteristic of the carbonyl 1,4-pirone system, and signals in the $\delta_{\mathrm{C}} 122.0-165.3$ region, attributed to ring $\mathrm{B}$ carbons, were observed. The signals at $\delta_{\mathrm{C}} 104.9$ and 165.3, corresponding to carbons $\mathrm{C}-3$ and $\mathrm{C}-4$ of ring $\mathrm{C}$, are typical of flavone aglycone. These data were compared with data in the literature (Khadem and Marles 2010) and were consistent for tricin.

The ethyl acetate extract from young leaves and culms of $S$. officinarum and the hexane, ethyl acetate and methanol partitions from the ethyl acetate extract were tested at concentrations ranging from 0.25 to $250 \mu \mathrm{g} / \mathrm{mL}$ against 08 different human tumor cell lines [glioma (U521), breast (MCF-7), resistant ovary (NCI/ADR-RES), kidney (786-0), lung (NCI-H460), prostate (PC3), ovary (OVCAR-3) and colon (HT29)] and nontumoral human keratinocyte (HaCat) cell lines using a colorimetric method for growth inhibition evaluation (Table I). Doxorubicin was used as a positive control.

Human cell lines: A = U251 (glioma, SNC); B $=$ MCF-7 (breast); $\mathrm{C}=$ NCI-ADR/RES (multiple drug resistant ovary cells); $\mathrm{D}=786-0$ (kidney); $\mathrm{E}=$ NCI-H460 (lung); F = PC-3 (prostate); $\mathrm{G}=$ OVCAR-3 (ovary); H = HT29 (colon); Q = HaCat (normal human keratinocytes). *Doxorubicin: reference chemotherapy; $\mathrm{GI}_{50}$ : Concentration necessary for $50 \%$ cell growth inhibition.

In general, the ethyl acetate extract showed cytostatic activity in concentrations ranging from 25.8 to $61.8 \mu \mathrm{g} / \mathrm{mL}$. Similar activity was noticed for the ethyl acetate partition, suggesting that the compounds responsible for this biological activity were concentrated in this fraction. Corroborating these results, the hexanic and methanolic fractions 
TABLE I

$\mathrm{GI}_{50}(\mu \mathrm{g} / \mathrm{mL})$ for the $S$. officinarum crude ethyl acetate extract and partitions for cancer cell lines.

\begin{tabular}{lccccccccc}
\hline & $\mathbf{A}$ & $\mathbf{B}$ & $\mathbf{C}$ & $\mathbf{D}$ & $\mathbf{E}$ & $\mathbf{F}$ & $\mathbf{G}$ & $\mathbf{H}$ & $\mathbf{Q}$ \\
\hline Doxorubicin * & $<0.025$ & $<0.025$ & 0.025 & 0.20 & $<0.025$ & 0.082 & 0.28 & 0.12 & 0.025 \\
\hline EtAc extract & 29.0 & 25.8 & 25.4 & 31.8 & 28.7 & 47.8 & 57.9 & 61.8 & 26.1 \\
\hline Hexane part. & $>250$ & 164.0 & 162.5 & $>250$ & $>250$ & $>250$ & $>250$ & $>250$ & $>250$ \\
\hline EtAc part. & 29.1 & 26.1 & 5.9 & 65.4 & 51.7 & 30.3 & 52.4 & 35.0 & 34.2 \\
\hline Methanol part. & $>250$ & 67.2 & $>250$ & $>250$ & $>250$ & $>250$ & $>250$ & $>250$ & $>250$ \\
\hline pure tricin & $>250$ & $>250$ & 70.3 & $>250$ & 127.7 & $>250$ & 41.1 & $>250$ & 69.6 \\
\hline
\end{tabular}

showed a comparatively lower activity, being inactive $\left(\mathrm{GI}_{50}>250 \mu \mathrm{g} / \mathrm{mL}\right)$ against almost all cell lines (Table I). Thus, fractionation of the ethyl acetate fraction was prioritized.

Of the compounds isolated from the ethyl acetate fraction, flavonoid tricin was evaluated against the same cell line panel. Tricin showed cytostatic activity against two ovarian cancer cell lines, OVCAR-3 $\left(\mathrm{GI}_{50}=41.1 \mu \mathrm{g} / \mathrm{mL}\right)$ and NCIADR $/$ RES $\left(\mathrm{GI}_{50}=70.3 \mu \mathrm{g} / \mathrm{mL}\right)$. As observed for doxorubicin, tricin also inhibited cell growth of non tumoral human keratinocytes (HaCat, $\mathrm{GI}_{50}=$ $69.6 \mu \mathrm{g} / \mathrm{mL}$ ). Therefore, this result partly explains the biological activity observed for the ethyl acetate crude extract and the ethyl acetate fraction.

Based on literature results (Kampa et al. 2003, Lee 2005, Pugazhendhi et al. 2005) for the other phenolic compounds isolated from the bioactive ethyl acetate fraction ( $p$-hydroxybenzoic, $p$-hydroxycinnamic, vanillic and ferulic acid), it is possible to postulate the involvement of these compounds in the cytostatic activity observed for the ethyl acetate crude extract and the ethyl acetate fraction. (Cai et al. 2004) evaluated tricin in a xenographic breast tumor model and observed that treating animals with tricin $(0.2 \%$ $\mathrm{w} / \mathrm{w}$ in diet) for 7 days before tumor cell (MDAMB-468) implantation did not reduce tumor growth, but tricin treatment $(72 \mathrm{~h}, 11 \mu \mathrm{M})$ before
MDA-MB-468 tumor cell implantation in nude mice resulted in smaller tumors when compared to untreated cells. Besides, extracts containing high tricin concentrations are known to inhibit human colon and breast cancer cell proliferation (Hudson et al. 2000). There is also evidence that tricin has chemopreventive activity, such as observed in intestinal carcinogenesis models in mice (Cai et al. 2005).

The phytochemical study of the leaves and culms of Saccharum officinarum led to the isolation of eleven compounds belonging to different classes. Their structures were identified based on GC-MS and NMR methods. The tricin showed significant anticancer activity in the in vitro anticancer assay. Therefore, the present isolation of tricin corroborates anticancer studies, pointing to the optimization of the production of tricin from this new source for important pharmacological studies. Sugarcane may also be an important source of tricin derivatives which may be used to produce the bioactive aglycone by hydrolysis.

\section{ACKNOWLEDGMENTS}

The authors thank Conselho Nacional de Desenvolvimento Científico e Tecnológico (CNPq), Coordenação de Aperfeiçoamento de Pessoal de Nível Superior (CAPES) and Fundação Araucária for their financial support. 


\section{REFERENCES}

ABBAS SR, AHMAD SD, SABIR AH, SHAH AH, AWAN S, GOHAR M, KHAN MF AND RAO AZ. 2013. Antioxidant activity, repair and tolerance of oxidative DNA damage in different cultivars of Sugarcane (Saccharum officinarum) leaves. J Crop Sci 7: 40-45.

AHMAD N ET AL. 2011. DPPH-scavenging antioxidant potential in regenerated tissues of Stevia rebaudiana, Citrus sinensis and Saccharum officinarum. J Med Plant Res 5: 3293-3297.

ALMEIDA JMD, NEGRI G, SALATINO A, CARVALHO JE AND LAJOLO FM. 2007. Antiproliferative and antioxidant activities of a tricin acylated glycoside from sugarcane (Saccharum officinarum) juice. Phytochemistry 68: 1165-1171.

BARROS FMC, PEREIRA KN, ZANETTI GD AND HEINZMANN BM. 2007. Plantas de uso medicinal no município de São Luiz Gonzaga, RS, Brasil. Lat Am J Pharm 26: 652-662.

CAI H, HUDSON EA, MANN P, VERSCHOYLE RD, GREAVES P, MANSON MM, STEWARD WP AND GESCHER AJ. 2004. Growth-inhibitory and cell cyclearresting properties of the rice bran constituent tricin in human-derivated breast cancer cells in vitro and in nude mice in vivo. Br J Cancer 91: 1364-1371.

CAI H, STEWARD WP AND GESHER AJ. 2005. Determination of the putative cancer chemopreventive flavones tricin in plasma and tissues of mice by HPLC with UV-visible detection. Biomed Chrom 19: 518-522.

CHATTERJEE A AND PAKRASHI SC. 1995. The treatise on Indian Medicinal plants. Volume 1 to 5. New Delhi: Publications and Information Directorate; India.

CHUNG IM, HAHN SJ AND AHMAD A. 2005. Confirmation of potential herbicidal agents in hulls of rice, Oryza sativa. J Chem Ecol 31: 1339-1352.

COLOMBO R, LANÇAS FM AND YARIWAKE JH. 2006 b. Determination of flavonoids in cultivated sugarcane leaves, bagasse, juice and in transgenic sugarcane by liquid chromatography-UV detection. J Chromatogr 1103: 118-124.

COLOMBO R, YARINWAKE JH, QUEIROZ EF, NDJOKO K AND HOSTETTMANN K. 2005. On-line identification of sugarcane (Saccharum officinarum L.) methoxyflavones by liquid chromatography-UV detection using postcolumn derivatization and liquid chromatography-mass spectrometry. J Chromatogr 1082: 51-59.

COLOMBO R, YARIWAKE JH, QUEIROZ EF, NDJOKO K AND HOSTETTMANN K. 2006a. On-line identification of futher flavones $\mathrm{C}$ - and O-glycosides from sugarcane (Saccharum officinarum L., Gramineae) by HPLC-UVMS. Phytochem Anal 17: 337-343.
CONAB - COMPANHIA NACIONAL DE ABASTECIMENTO ACOMPANHAMENTO DA SAFRA BRASILEIRA CANADE-AÇÚCAR. 2013. Disponível em: <http://www.conab. gov.br/OlalaCMS/uploads/arquivos/13_08_08_09_39_29_ boletim cana portugues - abril 2013 1o lev.pdf.>. Acesso: Julho, 2015.

DUARTE-ALMEIDA JM, SALATINO A, GENOVESE MI AND LAJOLO FM. 2011. Phenolic composition and antioxidant activity of culms and sugarcane (Saccharum officinarum L.) products. Food Chem 125: 660-664.

GOULART MOF, SANT'ANA AEG, LIMA RA AND CALVACANTE S. 1993. Fitoconstituintes químicos isolados de Jatropha elleptica. Atribuição dos deslocamentos químicos dos átomos de carbono e hidrogênio dos diterpenos jatrofolanos A e B. Quím Nova 16: 95-100.

HUDSON EA, DINH PA, KOKUBU T, SIMMONDS MS AND GESCHER A. 2000. Characterization of potentially chemopreventive phenols in extracts of brown rice that inhibit the growth of human breast and colon cancer cells. Cancer Epidemiol Biomarkers Prev 9: 1163-1170.

KAMPA M ET AL. 2003. Antiproliferative and apoptotic effects of selective phenolic acids on T47D human breast cancer cells: potential mechanisms of action. Breast Cancer Res 6: 63-74.

KHADEM S AND MARLES RJ. 2010. Review Monocyclic Phenolic Acids; Hydroxy- and Polyhydroxybenzoic Acids: Occurrence and Recent Bioactivity Studies. Molecules 15: 7985-8005.

LEE JH, LEE JY, PARK JH, JUNG HS, KIM JS, KANG SS, KIM YS AND HAN Y. 2007. Immunoregulatory activity by daucosterol, a beta-sitosterol glycoside, induces protective Th1 immune response against dissemined Candidiasis in mice. Vaccine 25: 3834-3840.

LEE YS. 2005. Role of NADPH oxidase-mediated generation of reactive oxygen species in the mechanism of apoptosis induced by phenolic acids in HepG2 human hepatoma cells. Arch Pharm Res 28: 1183-1189.

LÓPEZ-LÁZARO M. 2009. Distribution and biological activities of the flavonoid luteolin. Mini Rev Med Chem 9: 31-59.

LOYD HLAND NAIDOO G. 1983. Chemical array potentially suitable for determination of smut resistence of sugarcane cultivars. Plant Dis 67: 1103-1105.

MIRANDA MLD, SOUZA AF, RODRIGUES ED, GARCEZ RF AND GARCEZ SWS. 2012. Constituintes Químicos das Folhas de Riedeliella graciliflora Harms (Leguminosae). Quím Nova 35: 1306-1311.

MONKS A ET AL. 1991. Feasibility of a high-flux anticancer drug screen using a diverse panel of cultured human tumor cell lines. J Natl Cancer Inst 83: 757-766.

PALLAVI R, ELAKKIYA S, TENNETY SSR AND DEVI PS. 2012. Anthocyanin analysis and its anticancer 
property from sugarcane (Saccharum officinarum L.) pee. International J Res Pharm Chem 2: 338-345.

PERRICONE NV AND DINARDO JC. 1996. Photoprotective and antiinflammatory effects of topical glycolic acid. Dermatol Surg 22: 435-437.

PUGAZHENDHI D, POPE GS AND DARBRE PD. 2005. Oestrogenic activity of p-hydroxybenzoic acid (common metabolite of paraben esters) and methylparaben in human breast cancer cell lines. J Appl Toxicol 25: 301-309.

RUCIANI L. 1997. Glycolic acid peel. J Eur Acad Dermat Venerol 9: 12-13.

SAJJADI SE, SHOKOOHINIA Y AND MOAYEDI NS. 2012. Isolation and identification of ferulic acid from aerial parts of Kelussia odoratissima Mozaffi. Jundishapur J Nat Pharm Prod 7: 159-162.

SCOTT KN. 1972. Carbon-13 nuclear magnetic resonance of biologically important aromatic acids. Chemical shifts of benzoic acid and derivatives. J Am Chem Soc 94: 85648568.

SHOEMAKER RH. 2006. The NCI 60 human tumor cell line anticancer drug screen. Nat Rev Cancer 6: 813-823.

SILVA AMS, ALKORTA I, ELGUERRO J AND SILVA VLM. 2001. A ${ }^{13} \mathrm{C}$ NMR study of the structure of four cinnamic acids and their methyl esters. J Mol Struct 595: 1-6.
TAKAHASHI M, KONNO C AND HIKINO H. 1985. Isolations and hypoglycemic activity of saccharans A, B, C, D, E and F, glycans of Saccharum officinarum stalks. Planta Med 51: 258-260.

VILA FC, COLOMBO R, LIRA TO AND YARIWAKE JH. 2008. HPLC microfractionation of flavones and antioxidant (radical scavenging) activity of Saccharum officinarum L. J Braz Chem 19: 903-908.

WALFORD S, EGLIS S AND MARTINCIGH B. 2009. Separation, identification and characterization of extractives from sugarcane (Saccharum officinarum) Leaf. Proc S Afr Sug Technol Ass 82: 342-345.

WILLIAMS CA, HARBORNE JB AND SMITH P. 1974. The taxonomic significance of leaf flavonoids in Saccharum and related genera. Phytochemistry 13: 1141-1149.

YAMASHITA F, SUZUKI T AND KESYOU I. 1993. Active compounds in cane molasses having effect on human skin. Proceedings of the Research Society of Japan Sugar Refineries Technologists 41: 43-48.

YU Y, GAO H, TANG Z, SONG X AND WU L. 2006. Several phenolic acids from the fruit of Capparis spinosa. Asian $\mathrm{J}$ Trad Med 1: 1-4. 